\title{
ELECTRIC FIELDS CREATED BY POINT CHARGES: SOME GEOMETRICAL AND TOPOLOGICAL RESULTS
}

\author{
ALBERTO ENCISO and DANIEL PERALTA-SALAS
}

Communicated by Gerald A. Goldin

Abstract. We study some geometrical and topological properties of the electric fields created by point charges on Riemannian manifolds. Particularly, we characterize the spaces on which the electric lines emanating from a point charge are geodesics, and describe the topological properties of the basin boundary for $N$ point charges. Several open problems will be posed.

\section{Introduction}

The discovery of the inverse-square law for Newtonian and Coulomb interactions is a milestone in the Physics of the XVII and XVIII centuries. The central claim $[1,14]$ is that, for both electric and gravitational interactions, the force per unit mass or charge experimented by a test particle situated at a point $\boldsymbol{x} \in \mathbb{R}^{3}$ is given by the field

$$
\boldsymbol{E}(\boldsymbol{x})=\frac{q}{4 \pi} \frac{\boldsymbol{x}-\boldsymbol{p}}{|\boldsymbol{x}-\boldsymbol{p}|^{3}} .
$$

Here $q \in \mathbb{R}$ is the charge (or minus the mass) of the point particle originating the interaction, $\boldsymbol{p} \in \mathbb{R}^{3}$ is its position and we have chosen Heaviside-Lorentz units.

Since then, the study of the electrostatic fields generated by $N$ point charges $q_{i}$ $(i=1, \ldots, N)$ in Euclidean space has become a classical problem in mathematical physics and potential theory [6]. When all the charges are negative, this is equivalent to studying the Newtonian field created by $N$ point masses $-q_{i}$. In modern treatments, one usually defines the potential function $V: \mathbb{R}^{3} \rightarrow \mathbb{R}$ of a unit point charge, which is a fundamental solution of the Poisson equation

$$
-\Delta V=\delta_{p}
$$

and obtains the electric field as $\boldsymbol{E}=-\nabla V$. Here $\delta_{p}$ stands for the Dirac distribution centered at $p$. The field of several charges can be calculated using the superposition principle. 
A natural generalization of this problem is the study of electrostatic fields on Riemannian spaces. In Theoretical Physics, this problem arises when studying static solutions to Maxwell's equations

$$
\mathrm{d} F=0, \quad \delta F=J
$$

in spacetimes of the form $(\tilde{M}, \tilde{g})=(M, g) \times\left(\mathbb{R},-\mathrm{d} t^{2}\right)$. Here $F$ is the field two-form and $J$ is the current one-form. Poisson's equation

$$
-\Delta V=\rho
$$

where $\Delta$ is the Laplace-Beltrami operator of the spatial manifold $(M, g)$, is readily obtained using the ansatz

$$
F=\mathrm{d} A, \quad A=-V \mathrm{~d} t, \quad J=-\rho \mathrm{d} t, \quad V, \rho \in C^{\omega}(M) .
$$

Equation (1) is usually tackled via fundamental solutions, which involve the analysis of the electric fields generated by static point charges on the space manifold $(M, g)$.

There is a vast literature on the fundamental solutions of the Poisson equation on manifolds, e.g., on the existence of positive fundamental solutions $[4,10,17,18$, $21]$, the study of upper and lower estimates for these functions [12, 19,24], and the connection of these fundamental solutions with the heat kernel $[11,20,26]$. Nevertheless, the geometric properties of the gradient of the fundamental solutions, i.e., the orbits of the electric fields created by a point charge, have received comparatively little attention. In this paper we will focus on the study of this aspect from the viewpoint of the theory of dynamical systems. Thus the concept of electric line, as Faraday used to visualize the electric fields in the XIX century, is extended to the framework of general Riemannian spaces. As a spin-off, interesting connections between the structure of the electric lines and the topology of the manifold will be shown.

In the next section we will state several geometrical and topological properties of the orbits of the electric field. To the best of our knowledge, both the results and the approach are new in the literature. The proofs of these statements will be published elsewhere [7]. The paper finishes with a section discussing some open problems.

\section{Statement of Results}

Let $(M, g)$ be an open Riemannian $n$-manifold without boundary, which we will assume to be complete, analytic, connected, finitely generated (i.e., all the homo- 
topy groups of $M$ have finite rank) and such that all its ends are collared. For an arbitrary point $p \in M$, let $V_{p}$ be a fundamental solution of the Poisson equation

$$
-\Delta V_{p}=\delta_{p}
$$

$\Delta$ standing for the Laplace-Beltrami operator. Li and Tam [17] provided a geometric construction of symmetric decreasing solutions of this equation for any Riemannian manifold $(M, g)$. Their technique consisted in considering a sequence of solutions $\left(V_{p}^{k}\right)_{k=1}^{\infty}$ of the Dirichlet problem

$$
\begin{aligned}
-\Delta V_{p}^{k} & =\delta_{p} & & \text { in } M_{k} \\
V_{p}^{k} & =0 & & \text { on } \partial M_{k}
\end{aligned}
$$

in an exhaustion of $M$ by compact sets $p \in M_{1} \subset M_{2} \subset \cdots$. Li and Tam's solution of equation (2), which is essentially unique in certain cases, can be obtained as

$$
V_{p}(x)=\lim _{k \rightarrow \infty} V_{p}^{k}(x)-c_{k}
$$

for some sequence of non-negative constants $\left(c_{k}\right)$, and is analytic in $M \backslash\{p\}$.

When inf $V_{p}=-\infty, V_{p}$ is called a non-positive Green function, or an Evans function. This property only depends on the end structure of $(M, g)$, not on the point $p \in M$, and in this case the Riemannian manifold is said to be parabolic. Otherwise, $V_{p}$ is called a (positive) Green function, and $(M, g)$ is hyperbolic. This terminology, which is standard in potential theory, is not related to the signature of the Riemannian metric on $M$, which is always positive definite. There is extensive literature on geometric conditions characterizing hyperbolic and parabolic spaces, e.g., [4, 10, 12, 19].

A configuration of point charges on $M$ is a set $C=\left\{\left(q_{i}, p_{i}\right)\right\}_{i=1}^{N}$, where $N$ is the number of charges, and $\left(q_{i}, p_{i}\right) \in(\mathbb{R} \backslash\{0\}) \times M$ represents the magnitude and position of the $i$-th charge of the configuration.

Definition 1. The electric field $\boldsymbol{E}$ created by the charge configuration $C$ is defined as $\boldsymbol{E}=-\nabla V$, whence the potential function $V$ is given by

$$
V=\sum_{i=1}^{N} q_{i} V_{p_{i}}
$$

$V_{p}$ standing for a solution to equation (2) obtained via Li and Tam's procedure. 
The electric field is obviously an analytic, divergence-free vector field on the manifold $M \backslash\left\{\bigcup_{i=1}^{N} p_{i}\right\}$, and satisfies Maxwell's equations there. Observe that the definition of the electric field does not require $(M, g)$ to be hyperbolic. In fact, since we will be interested in the properties of the orbits of $E$ (which from now on will be called electric lines), the hyperbolicity or parabolicity of the manifold will not be especially relevant. Actually, recall that even the Euclidean plane $\left(\mathbb{R}^{2}, \delta\right)$ is a parabolic space. One should also note that Li and Tam's solutions to equation (2) are physically admissible in both cases, since they are symmetric and decreasing, which are in fact the only properties of the potential that will be used in this article.

The analytical local behavior of the electric field near a point charge is well known [9]. Calling $r$ the geodesic distance to a charge situated at $p_{i}$, there exists a real constant $c$ such that

$$
\boldsymbol{E}=\frac{c}{r^{n-1}} \partial_{r}+O\left(\frac{1}{r^{n-3}}\right)
$$

in a neighborhood of $p_{i}$. However, the topological structure of the electric field is not well understood as a dynamical system, neither locally (e.g., portrait near the charges and the zeros of the electric field) nor its global aspects (e.g., topology of the attracting or repelling basins).

Let us concentrate on these topological issues. In the following theorem we will gather several general properties of the electric lines.

Theorem 2. For the electric field $\boldsymbol{E}$ created by the charge configuration $C$, the following statements hold:

1. $p_{i}$ is a local attractor or repeller, so its neighboring equipotential hypersurfaces $V^{-1}(c)(c \in \mathbb{R})$ are topological spheres.

2. (Analytic finiteness.) In a neighborhood of $p_{i}$, the electric trajectories are non-oscillating, i.e., if $A$ is an analytic subset of $M$ then the electric lines either stay in $A$, or intersect it in a finite number of points. Hence all the orbits have well defined unit tangent at $p_{i}$.

3. If $q_{i}<0$ for all $i$, the electric trajectories point inward at the infinity (more precisely, at any end $\mathcal{E}$ of $M)$. In other terms, the ends of the manifold are local maxima and the equipotential hypersurfaces "near" each end are "tubes" around it.

4. There exist no invariant closed sets without charge with non-empty interior.

5. E cannot have any closed orbits, but it can have saddle connections. 
Properties 1 and 2 provide a quite detailed description of the electric lines near the charges modulo analytic (local) diffeomorphisms. The existence of a well-defined unit tangent along an orbit of a gradient vector field as one approaches a critical point, as in Property 2, has been extensively studied in the context of analytic dynamics [16,23]. Properties 3,4 and 5 provide information on the portrait of the electric lines in the large.

From now on, we will assume that all the charges are negative, i.e., $q_{i}<0$. Thus in this case the electric field can be also interpreted as the Newtonian gravitational field created by $N$ point masses of magnitude $-q_{i}$ and situated at $p_{i}$, which also coincides with the first order approximation to the gravitational field in General Relativity [22].

Definition 3. The (attracting) basin associated to the $i$-th charge is defined as

$$
D_{i}=\left\{x \in M ; \omega(x)=p_{i}\right\}
$$

where $\omega(x)$ denotes the $\omega$-limit of the orbit of $\boldsymbol{E}$ passing through $x$. The (attracting) basin of $C$ is

$$
D=\bigcup_{i=1}^{N} D_{i} .
$$

We will call $\mathcal{F}=\partial D$ the basin boundary of $C$.

Thus $D_{i}$ consists of the electric lines falling into $p_{i}$, and the boundary is composed of points which are not dragged into any charge along $E$. It can be proved that $D_{i}$ is an open, invariant submanifold of $M$ diffeomorphic to $\mathbb{R}^{n}$, and therefore $D$ and $\mathcal{F}$ are disjoint.

In the following theorem we provide a topological characterization of the basin boundary.

Theorem 4. The following statements hold:

1. $\mathcal{F}$ is closed and invariant.

2. $M=D \cup \mathcal{F}$.

3. $1 \leq \operatorname{dim} \mathcal{F} \leq n-1$.

4. $\mathcal{F} \neq \emptyset$ if $M \neq \mathbb{R}^{n}$ or $N>1$.

5. $\mathcal{F}$ consists of the union of the zeros of $E$ and their stable components. 
Property 5 describes how the stable components of the critical points of $E$ generate the basin boundary. Property 2 implies that $M \backslash\{\mathcal{F}\}$ is diffeomorphic to $N$ disjoint copies of $\mathbb{R}^{n}$. When $N=1$, removing $\mathcal{F}$ from $M$ makes the manifold diffeomorphic to $\mathbb{R}^{n}$, and this suggests that the boundary should contain some information on the topology of the space, as the following theorem shows. Here $\mathcal{E}(M)$ denote the set of ends of $M$, and $\hat{M}=M \cup \mathcal{E}(M)$ is the collared end compactification of $M$ [13], which is also a topological manifold since $M$ is finitely generated. Furthermore, we shall denote $\hat{\mathcal{F}}=\mathcal{F} \cup \mathcal{E}(M)$.

Theorem 5. For each $k<n-1$, the $k$-th homotopy group $\pi_{k}(\hat{M})$ (respectively homology group $\left.H_{k}(\hat{M})\right)$ of the compactified space is isomorphic to the $k$-th shape group $\check{\pi}_{k}(\hat{\mathcal{F}})$ (respectively Čech homology group $\left.\check{H}_{k}(\hat{\mathcal{F}})\right)$ of the compactified basin boundary. Furthermore, there exists a monomorphism $\pi_{n-1}(\hat{M}) \rightarrow$ $\check{\pi}_{n-1}(\hat{\mathcal{F}})$, and the groups $H_{n-1}(\hat{M}) \oplus \mathbb{Z}^{N-1}$ and $\check{H}_{n-1}(\hat{\mathcal{F}})$ are isomorphic.

An interesting observation is that this result would allow an observer to obtain the homology groups (up to order $n-1$ ) of the physical space $M$ by measuring the points where the electric field vanishes and following the incoming electric lines. One should recall $[2,5]$ that the singular homology (respectively homotopy) groups and Čech homology (respectively shape) groups are isomorphic for absolute neighborhood retracts, e.g. topological manifolds.

From now on we will assume $N=1$, and that the only charge is situated at $p$ and of magnitude -1 . In this case, the topological properties of the basin boundary resemble those of the cut locus $C(p)$ of $(M, g)$ at $p$. Thus we will now focus on the relationship between the cut locus and the boundary, and the geodesics and the electric lines.

Theorem 6. There exists an analytic diffeomorphism $D \rightarrow M \backslash\{C(p)\}$ mapping the electric lines into the geodesics starting at p.

However, the boundary is not generally homeomorphic to the cut locus, and the electric lines do not coincide with the geodesics. Therefore, the electric lines arise as a new kind of curves with geometrical meaning on a Riemannian manifold.

In the following theorem we provide the necessary and sufficient condition for the electric lines to be geodesics.

Theorem 7. The electric lines emanating from $p$ are geodesics if and only if the cut locus of $p$ is empty and $(M, g)$ is harmonic with respect to $p$. 
Recall a space is harmonic [25] with respect to $p$ when the volume density function in normal Riemann coordinates centered at $p$ depends only on the geodesic distance to $p$.

Example 8. Under the hypotheses of Theorem 7, the electric field is given by

$$
\boldsymbol{E}=\frac{c_{n}}{\sqrt{G} r^{n-1}} \partial_{r}
$$

where $r$ denotes the geodesic distance to $p, G$ is the determinant of the metric in normal Riemann coordinates, and $c_{n}^{-1}$ is the area of the unit round $(n-1)$-sphere. For instance, in the hyperbolic space $\mathbb{H}^{n}$

$$
\boldsymbol{E}=\frac{c_{n}}{\sinh ^{n-1} r} \partial_{r}
$$

\section{Open Problems}

A major open problem is to establish whether the electric field created by an arbitrary configuration of point charges is generically Morse-Smale, i.e., the critical points and periodic orbits are hyperbolic, the stable manifold of a critical element and the unstable manifold of another critical element intersect transversally, and the non-wandering set only consists of critical points and periodic orbits. It can be proved that this is the case in $\left(\mathbb{R}^{2}, g\right)$, but otherwise the problem is still open. Morse-Smale electric fields are structurally stable, and hence small perturbations of the magnitudes or positions of the charges would not modify the portrait of the electric lines for generic configurations.

Even in the Euclidean space $\mathbb{R}^{n}$ there are important unsolved questions. For instance, Eremenko [8] offers $\$ 200$ for the following problem: Does the electric field

$$
\boldsymbol{E}=\sum_{i=1}^{\infty} q_{i} \frac{\boldsymbol{x}-\boldsymbol{p}_{i}}{\left|\boldsymbol{x}-\boldsymbol{p}_{i}\right|^{n}}
$$

vanish somewhere for any infinite configuration of positive point charges $C=$ $\left\{\left(q_{i}, \boldsymbol{p}_{i}\right)\right\}_{i=1}^{\infty}$ in $\mathbb{R}^{n}$ satisfying certain convergence conditions?

Another open question is the celebrated Morse-Cairns problem [3]: Is there a configuration of positive point charges $C=\left\{\left(q_{i}, \boldsymbol{p}_{i}\right)\right\}_{i=1}^{N}$ in $\mathbb{R}^{3}$ such that the electric field

$$
\boldsymbol{E}=\sum_{i=1}^{N} q_{i} \frac{\boldsymbol{x}-\boldsymbol{p}_{i}}{\left|\boldsymbol{x}-\boldsymbol{p}_{i}\right|^{3}}
$$


vanishes on a curve? It is not difficult to check that the critical points of the electric field on a two-manifold are always isolated as a consequence of CauchyKowalewski's theorem, but the three dimensional case has proved to be much harder. It is known [15] that such critical curves (which must be compact and without endpoints for analyticity reasons) can appear when one allows positive and negative charges. We would happily pay another $\$ 200$ to anyone providing a full solution to this problem.

\section{Acknowledgements}

AE acknowledges the financial support of the MEC through an FPU scholarship, and the partial financial support by the DGI under grant \# BFM2002-02646.

\section{References}

[1] Bamberg P. and Sternberg S., A Course in Mathematics for Students of Physics, Cambridge University Press, Cambridge, 1990.

[2] Borsuk K., Theory of Shape, Polish Scientific Publishers, Warsaw, 1975.

[3] Cairns S. and Morse M., Critical Point Theory in Global Analysis and Differential Topology, Academic Press, New York, 1969.

[4] Cheng S. and Yau S., Differential Equations on Riemannian Manifolds and their Geometric Applications, Comm. Pure Appl. Math. 28 (1975) 333-354.

[5] Cordier J. and Porter T., Shape Theory, Ellis Horwood, Chichester, 1989.

[6] Courant R. and Hilbert, D., Methods of Mathematical Physics, Wiley, New York, 1989.

[7] Enciso A. and Peralta-Salas D., Geometric and Topological Aspects of Electrostatics on Riemannian Manifolds, Preprint.

[8] Eremenko A., http: //www. math.purdue. edu/ reremenko/.

[9] Gilbarg D. and Serrin J., On Isolated Singularities of Solutions of Second Order Elliptic Differential Equations, J. Anal. Math. 4 (1955/56) 309-340.

[10] Grigoryan A., On the Existence of a Green's Function on a Manifold, Russ. Math. Surv. 38 (1983) 190-191.

[11] Grigoryan A. and Saloff-Coste L., Dirichlet Heat Kernel in the Exterior of a Compact Set, Comm. Pure Appl. Math. 55 (2002) 93-133.

[12] Holopainen I., Volume Growth, Green's Functions, and Parabolicity of Ends, Duke Math. J. 97 (1999) 319-346. 
[13] Hughes B. and Ranicki A., Ends of Complexes, Cambridge University Press, Cambridge, 1996.

[14] Jackson J., Classical Electrodynamics, Wiley, New York, 1999.

[15] Janusauskas A., Critical Points of Electrostatic Potentials, Differencialnye Uravnenija 97 (1971) 84-90.

[16] Kurdyka K., Mostowski T. and Parusinski A., Proof of the Gradient Conjecture of R. Thom, Ann. of Math. 152 (2000) 763-792.

[17] Li P. and Tam L., Symmetric Green's Functions on Complete Manifolds, Amer. J. Math. 109 (1987) 1129-1154.

[18] Li P. and Tam L., Harmonic Functions and the Structure of Complete Manifolds, J. Diff. Geom. 35 (1992) 359-383.

[19] Li P. and Tam L., Green's Functions, Harmonic Functions, and Volume Comparison, J. Diff. Geom. 41 (1995) 277-318.

[20] Li P., Tam L. and Wang J., Sharp Bounds for the Green's Function and the Heat Kernel, Math. Res. Lett. 4 (1997) 589-602.

[21] Malgrange B., Existence et Approximation des Solutions des Équations aux Dérivées Partielles et des Équations de Convolution, Ann. Inst. Fourier 6 (1955) 271-355.

[22] Misner C., Thorne K. and Wheeler J., Gravitation, W. H. Freeman and Company, New York, 2002.

[23] Moussu R., Sur la dynamique des gradients, Math. Ann. 307 (1997) 445460.

[24] Royden H., The Growth of a Fundamental Solution of an Elliptic Divergence Structure Equation. In: Studies in Mathematical Analysis and Related Topics, G. Szëgö et al. (Eds.), Stanford University Press, Stanford, 1962, pp. 333-340.

[25] Ruse H., Walter A. and Willmore T., Harmonic Spaces, Edizioni Cremonese, Rome, 1961.

[26] Varopoulos N., Green's Functions on Positively Curved Manifolds, J. Funct. Anal. 45 (1982) 109-118.

\author{
Alberto Enciso \\ Departamento de Física Teórica II \\ Universidad Complutense \\ 28040 Madrid, SPAIN \\ E-mail address: aenciso@fis.ucm.es
}


Daniel Peralta-Salas

Departamento de Física Teórica II

Universidad Complutense

28040 Madrid, SPAIN

E-mail address: dperalta@fis.ucm.es 